\title{
MUON ENERGY LOSSES AND STRAGGLING FOR HIGH ENERGY MUONS
}

\author{
B. P. ROE
}

The University of Michigan, Ann Arbor, Michigan 48104, U.S.A.

Received 16 November 1970

The non-Gaussian nature of energy loss straggling is examined. A1 NAL large muon shields must be designed to reduce flux by about a factor of $10^{12}$. Fortunately it is shown here that on the long range end, the straggling falls off much faster than Gaussian and the amount of shielding needed is significantly reduced.

\section{Introduction}

The purpose of this article is to look at range and straggling effects as they influence design of NAL muon shields. The major problem here is that one is trying to shield down to levels of around 1 in $10^{12}$. I will generally limit myself to collision losses and direct pair production only. Fluctuations on bremsstrahlung losses are known to be so large that they are useless to this kind of level. (The shield is $\approx 1$ muon radiation length.) Nuclear interactions should probably also be examined as the nuclear cross sections are dominated by low energy transfers, due to giant dipole resonance, and may be useable.

We are used to taking errors as Gaussian. However, if true this would be disastrous for shielding to this level. For the $10^{-12}$ region we want to go out some 7 standard deviations. Since at NAL energies $(A R / R)^{2}$ is typically $10 \%$ one would have to add $70 \%$ to the calculated range.

Fortunately as we shall see the errors are not Gaussian and a much smaller number suffices.

\section{Source of straggling}

To begin with we will consider collision loss only, ignoring the effects of direct pair production. Let us first ask what fraction of the energy loss is due to high transferred energies. We use the results quoted by Rossi ${ }^{1}$ ) modified slightly for spin $\frac{1}{2}$ particles:

$\frac{\mathrm{d} E}{\mathrm{~d} x}\left(\mathrm{gcm}^{2}\right)=\frac{2 C m_{\mathrm{c}}}{\beta^{2}}$

where

$$
\times\left[\ln A-\beta^{2}\left(1+\frac{E_{\mathrm{MAx}}^{\prime}}{E_{\mathrm{m}}^{\prime}}\right)+\frac{1}{4} \frac{E_{\mathrm{MAX}}^{\prime 2}}{\left(E_{\mu}+m_{\mu}\right)^{2}}\right],
$$

$$
A=\frac{2 m_{\mathrm{e}} p_{\mu}^{2} E_{\mathrm{MAX}}^{\prime}}{m_{\mu}^{2} I^{2}(Z)}
$$

$E_{\mathrm{MAx}}^{\prime}=$ the maximum energy transfer we wish to

consider;

$$
\begin{aligned}
E_{\mathrm{m}}^{\prime}= & \text { the upper limit of } E_{\mathrm{MAX}}^{\prime}: \\
E_{\mathrm{m}}^{\prime}= & \frac{2 m_{\mathrm{e}} p_{\mu}^{2}}{m_{\mathrm{e}}^{2}+m_{\mu}^{2}+2 m_{\mathrm{c}} E_{\mu}} \\
& \left.\quad \text { for } E_{\mu} \gg 20 \mathrm{GeV}, E_{\mathrm{m}}^{\prime} \sim E_{\mu}\right) ; \\
I= & \text { ionization polential of the atom; } \\
C= & \pi N(\mathrm{Z} / A) r_{\mathrm{e}}^{2}=0.15(Z / A) .
\end{aligned}
$$

This formula ignores the density effect. The density effect only involves low energy transfers where the statistical fluctuation is small. The influence of this effect will be considered later.

The results are given in table 1 . We first note that over most of the range the probabilities of different percentages of energy loss remain approximately constant. This occurs since $A$ is such a huge number (around $10^{20}$ ) that $\ln A$ doesn't change by a large percentage even if $A$ changes by a factor of a hundred or so. The major cause of straggling is now clear. For instance look at the top 2 lines of table $1.2 .3 \%$ of the energy loss occurs because of collisions with more than $20 \%$ of the muon energy transferred. This is far less than 1 collision per stopping muon. The approximate numbers of collisions in each interval are given in the last column for $500 \mathrm{GeV}$ muons. Until transfer gets well below $1 \%$ of the incident energy one is dealing with statistics of very small numbers. However, this accounts for only around $10 \%$ of the energy loss and the rest is subject to much smaller fluctuations.

\section{Straggling due to collision loss}

We will now develop a straggling formula valid for that part of the collision loss caused by low energy transfers.

The energy loss in $\mathrm{d} x$ from collisions transferring between $E^{\prime}$ and $E^{\prime}+\mathrm{d} E^{\prime}$ is $E^{\prime} N\left(E^{\prime}\right) \mathrm{d} E^{\prime} \mathrm{d} x$ where $N=$ number of these collisions $\left(\mathrm{GeV} / \mathrm{gcm}^{2}\right)$. We approximate the error in this by taking the square root of the number of collisions i.e. $E^{\prime 2} N\left(E^{\prime}\right) \mathrm{d} E^{\prime} \mathrm{d} x=(\delta E)^{2}$. The total error is obtained by taking 
TABLE 1

Energy loss by collision. Fraction of energy loss due to large energy collisions.

\begin{tabular}{|c|c|c|c|c|c|}
\hline$E_{\mu}(\mathrm{GeV})$ & $E_{M A X}^{\prime}$ & $E_{\wedge \wedge \mathrm{N}}^{\prime} / E_{\mu}$ & $\ln A-\beta=\left(\frac{1+E_{\mathrm{MAX}}^{\prime}}{E_{\mathrm{m}}^{\prime}}\right)+\frac{1}{4} \frac{E_{M A \mathrm{X}}^{\prime}}{\left(E_{\mu}+m_{\mu}\right)^{2}}$ & $\begin{array}{l}\% \mathrm{~d} E / \mathrm{d} x \text { from energy } \\
\text { transfers }>E_{M A X}^{\prime}\end{array}$ & $\begin{array}{l}\text { No. of collisions in } \\
\text { interval }\end{array}$ \\
\hline \multirow[t]{7}{*}{500} & 500 & 1 & 44.95 & 0 & 0.045 \\
\hline & 100 & 0.2 & 43.9 & 2.3 & 0.08 \\
\hline & 50 & 0.1 & 43.4 & 3.5 & 0.7 \\
\hline & 10 & 0.02 & 41.8 & 7.0 & 1 \\
\hline & 5 & 0.01 & 41.1 & 8.6 & 7 \\
\hline & 1 & 0.002 & 39.5 & 12.1 & 10 \\
\hline & 0.5 & 0.001 & 38.8 & 13.7 & \\
\hline \multirow[t]{7}{*}{100} & 100 & 1 & 41.65 & 0 & \\
\hline & 20 & 0.2 & 40.6 & 2.6 & \\
\hline & 10 & 0.1 & 40.1 & 3.7 & \\
\hline & 2 & 0.02 & 38.5 & 7.6 & \\
\hline & 1 & 0.01 & 37.8 & 9.3 & \\
\hline & 0.2 & 0.002 & 36.2 & 13.1 & \\
\hline & 0.1 & 0.001 & 35.5 & 14.8 & \\
\hline
\end{tabular}

$$
\sum(\delta E)^{2}=(\Delta E)^{2}=\iint_{0}^{E_{\mathrm{MAx}}}\left(E^{\prime}\right)^{2} N\left(E^{\prime}\right) \mathrm{d} E^{\prime} \mathrm{d} x
$$

where $E_{\mathrm{MAX}}$ is the maximum energy transfer we wish to consider. We then approximate $\Delta R / R \approx \Delta E / E_{\mu}$ where $E_{\mu}$ is the initial energy.

For most of the range the energy loss is very crudely constant. (Again this occurs as $A$ is approximately constant, see table 1. The inclusion of the density effect only helps this approximation.)

Hence

We use ${ }^{1}$ )

$$
E=\overline{\left(\frac{\mathrm{d} E}{\mathrm{~d} x_{\text {total }}}\right)}(R-x), \quad \text { where } R=\text { range. }
$$

$$
\begin{aligned}
N_{\text {coll }}\left(E, E^{\prime}\right) \mathrm{d} E^{\prime}= & \frac{2 C m_{\mathrm{e}}}{\beta^{2}} \frac{\mathrm{d} E^{\prime}}{\left(E^{\prime}\right)^{2}} \\
& \times\left[1-\beta^{2} \frac{E^{\prime}}{E_{\mathrm{m}}^{\prime}}+\frac{1}{2}\left(\frac{E^{\prime}}{E+m_{\mu}}\right)^{2}\right] .
\end{aligned}
$$

(Note: the last term is specific to spin $\frac{1}{2}$ particles.) We ignore very low energy collisions as a source of straggling. (For these collisions $\left(E^{\prime}<\eta\right)$ atomic effects are important.) We also set $\beta=1$ and ignore $m_{\mu}$ compared to $E$. Then

$$
\begin{aligned}
\Delta E^{2} & =\iint_{\eta}^{E_{\mathrm{MAX}}^{\prime}} E^{\prime 2} N \mathrm{~d} E^{\prime} \mathrm{d} x \\
& =\frac{2 C m_{\mathrm{e}}}{\beta^{2}} \frac{E_{\mathrm{MAX}}^{\prime}}{2}\left(1-\frac{E_{\mathrm{MAX}}^{\prime}}{2 E^{\prime} m}+\frac{1}{6} \frac{E_{\mathrm{MAX}}^{\prime 2}}{E_{\mu}^{2}}\right) \frac{E_{\mu}}{(\mathrm{d} E / \mathrm{d} x)_{\mathrm{tot}}} .
\end{aligned}
$$

This derivation has assumed that at any point in the path we consider losses due to collisions below a constant fraction of the energy at that point. The fraction $F=\left(E_{\mathrm{MAX}}^{\prime} / E_{\mu}\right)_{\mathrm{original}}$. Using our previous expression for $\mathrm{d} E / \mathrm{d} x$ (including only collision losses and ignoring the density effect for now) we obtain:

$$
\begin{aligned}
\left(\frac{\Delta R}{R}\right)^{2}=\left(\frac{\Delta E}{E_{\mu}}\right)^{2}= & \frac{E_{\mathrm{MAX}}^{\prime}}{E_{\mu}}\left[2\left(\left\{\ln \frac{2 m_{\mathrm{e}} \bar{E}_{\mu}^{3}}{m_{\mu}^{2} I^{2}(Z)}\right\}-1.75\right)\right]^{-1} \\
& \times\left(1-\frac{E_{\mathrm{MAX}}^{\prime}}{2 E_{\mathrm{m}}^{\prime}}+\frac{1}{6} \frac{E_{\mathrm{MAX}}^{\prime 2}}{E_{\mu^{2}}}\right),
\end{aligned}
$$$$
E_{\mu}>20 \mathrm{GeV} .
$$

The density effect reduces the denominator somewhat increasing the error. At 500 (100) GeV $(\mathrm{d} E / \mathrm{d} x)_{\mathrm{no}}$ density effect $=3.17(2.82)$ and the Sternheimer ${ }^{2}$ ) density effect is for iron $0.88(0.65) .(\Delta R / R)^{2}$ above should then be multiplied by a factor 3.17 / $(3.17-0.88)=1.38$ et $500 \mathrm{GeV}$. The important thing to note here is that the error decreases as $E_{\mathrm{MAx}}^{\prime} / E_{\mu}=F$. For $F=1, \Delta R / R=0.087$ at $500 \mathrm{GeV}(0.10$ if density effect is included.)

Suppose we want to shield $500 \mathrm{GeV}$ muons to the $10^{-12}$ level. If we set $E_{\mathrm{MAX}}^{\prime} / E_{\mu}$ at 0.002 then (including density effect) $\Delta R / R \approx 0.0055$. Using 7 standard deviations we see $7 \times 0.55 \%=3.9 \%$ and we must also include the $16.4 \%^{3}$ ) of the loss due to high energy transfers. Hence we should use

$$
\frac{1-0.16}{1+0.039}\left(\frac{\mathrm{d} E}{\mathrm{~d} x}\right)_{\text {full col. looss }}=0.80\left(\frac{\mathrm{d} E}{\mathrm{~d} x}\right)_{\mathrm{full} \text { col. lows }}
$$

for designing shielding to be safe if only collision losses are included. 
TABLE 2

Direct pair production in earth as a function of muon energy $(\mathrm{GeV})$ and $\alpha$.

\begin{tabular}{cccc}
\hline$E_{\mu}(\mathrm{GeV}) / \alpha$ & 1 & 1.5 & 3 \\
\hline & & & \\
100 & 0.17 & 0.19 & 0.22 \\
200 & 0.37 & 0.41 & 0.48 \\
300 & 0.58 & 0.64 & 0.74 \\
400 & 0.79 & 0.87 & 1.01 \\
500 & 1.00 & 1.10 & 1.28 \\
\hline
\end{tabular}

In passing we note that the above procedure can easily be modified for $E_{\mu}<20-40 \mathrm{GeV}$ and a closed form obtained for straggling at all relativistic energies. (This just involves replacing $E_{\mathrm{MAX}}$ by $E_{\mathrm{m}}^{\prime}$ and replacing the $\log$ by the appropriate terms with $E_{\mathrm{m}}^{\prime}<E_{\mu}$.)

There is a broad maximum in the straggling introduced by the fact that $E_{\mathrm{m}}^{\prime}$ approaches $E_{\mu}$ as $E_{\mu}$ increases. This has the effect of increasing the straggling due to more high energy collisions. However, the logarithm in the denominator also increases and eventually takes over. The maximum occurs at around 150 $\mathrm{GeV}(8.7 \%)$ if the density effect is ignored or $500 \mathrm{GeV}$ $(10 \%)$ if one includes the density effect.

\section{Straggling due to direct pair production}

Next we turn to direct pair production. The theory here is not in as good shape as that for collision losses and the existing formulas can only be integrated numerically. Most existing calculations ${ }^{4-7}$ ) are based on the work of Bhabha $^{8}$ ) and Racah $^{9}$ ) which are subject to several approximations. I will use the more modern treatment given by Murota, Ueda and Tanaka $\left.{ }^{10}\right)$. There is still much room for further improvement. A treatment using one of the computerized reductions of the matrix elements is clearly called for if the calculations are to get to $10 \%$ accuracy. In addition the atomic shielding has to be included in a better manner than at present. The formula I use is given in the appendix $\left.{ }^{11}\right)$. I use the completely shielded form whenever it gives a smaller result than the unshielded form. This should be a safe approximation since partial shielding should increase the energy loss over complete shielding.

There is a parameter, $\alpha$, in the treatment which is arbitrary but of the order of 1. Results for earth $\bar{Z}=12, \bar{Z} / A=0.5$ for different values of $\alpha$ give the results shown in table 2 . I settled on $\alpha \approx 1.5$ since this seemed to give in the logarithm $137 \alpha \approx 206$ which is close to the 183 used in many of the calculations.

Table 3 gives results for iron and table 4 gives results for earth (units are $\mathrm{MeV} / \mathrm{gcm}^{2}$ ). The last column in table 3 gives the results of a crude integration to get the average $(\mathrm{d} E / \mathrm{d} x)_{\mathrm{direct}}$ pair over the interval $0-500$ $\mathrm{GeV}$.

The values I obtain for $\mathrm{d} E / \mathrm{d} x$ are somewhat higher than those of Theriot ${ }^{7}$ ). For instance, at $500 \mathrm{GeV}$ on iron I find 2.03 while Theriot obtains 1.53 and at 100 GeV I obtain 0.35 while Theriot gets 0.275 .

Using the same procedure for $\Delta R / R$ as outlined for the collision loss case, but using numerical integration, I obtained the results shown in table 5 for $500 \mathrm{GeV}$ muons. Although not as good as collision loss again I find most of the energy is lost in low energy transfer collisions. The $R$ used for normalising here is the range from collision loss only. Fig. 1 shows some old results ${ }^{11}$ ) obtained at $200 \mathrm{GeV}$ for typemetal. The shape of the direct pair cross section can be seen here. It is clear that very low and very high energy transfers are both decreased relative to collision loss.

If we use $R$ from the total $\mathrm{d} E / \mathrm{d} x$ our errors in table 5 should be reduced by $1(2.3+0.98) / 2.3=1.20\left(\Delta R_{\mathrm{ncw}} /\right.$ $R=\Delta R_{\text {old }} / R / 1.20$ ).

TABLE 3

Direct pair production in iron as a function of muon energy $(\mathrm{GeV})$ and fractional energy transfer.

$\mathrm{d} E / \mathrm{d} x$ for energy transfers less than $E_{\mathrm{paur}}$

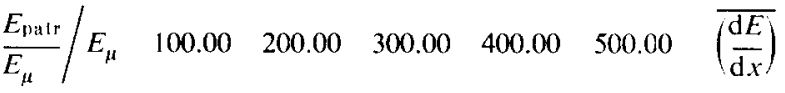

\begin{tabular}{lllllll}
\hline 0.0005 & 0.017 & 0.048 & 0.086 & 0.126 & 0.168 & 0.072 \\
0.0010 & 0.038 & 0.100 & 0.169 & 0.241 & 0.315 & 0.141 \\
0.0020 & 0.075 & 0.182 & 0.297 & 0.414 & 0.533 & 0.247 \\
0.0050 & 0.147 & 0.334 & 0.528 & 0.725 & 0.925 & 0.44 \\
0.0100 & 0.208 & 0.461 & 0.722 & 0.986 & 1.252 & 0.60 \\
0.0200 & 0.263 & 0.574 & 0.894 & 1.217 & 1.543 & 0.74 \\
0.0500 & 0.313 & 0.678 & 1.052 & 1.429 & 1.809 & 0.88 \\
0.1000 & 0.334 & 0.723 & 1.120 & 1.521 & 1.924 & 0.93 \\
0.2000 & 0.344 & 0.746 & 1.156 & 1.571 & 1.987 & 0.96 \\
1.0000 & 0.350 & 0.760 & 1.180 & 1.603 & 2.029 & 0.98
\end{tabular}

Fraction of $\mathrm{d} E / \mathrm{d} x$ due to energy losses greater than $E_{\text {palr }}$

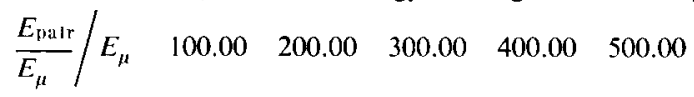

\begin{tabular}{llllll}
\hline 0.0005 & 0.951 & 0.936 & 0.927 & 0.921 & 0.917 \\
0.0010 & 0.891 & 0.868 & 0.857 & 0.850 & 0.845 \\
0.0020 & 0.785 & 0.760 & 0.749 & 0.742 & 0.737 \\
0.0050 & 0.581 & 0.561 & 0.553 & 0.548 & 0.544 \\
0.0100 & 0.406 & 0.394 & 0.388 & 0.385 & 0.383 \\
0.0200 & 0.249 & 0.245 & 0.242 & 0.241 & 0.240 \\
0.0500 & 0.106 & 0.108 & 0.109 & 0.109 & 0.109 \\
0.1000 & 0.047 & 0.050 & 0.051 & 0.051 & 0.052 \\
0.2000 & 0.017 & 0.019 & 0.020 & 0.020 & 0.021 \\
1.0000 & 0.000 & 0.000 & 0.000 & 0.000 & 0.000 \\
& & & & & \\
\hline
\end{tabular}


TABLE 4

Direct pair production in earth as a function of muon energy $(\mathrm{GeV})$ and fractional energy transfer.

$\mathrm{d} E / \mathrm{d} x$ for energy transfers less than $E_{\text {patr }}$

$\frac{E_{\text {palr }}}{E_{\mu}} / E_{\mu} \quad 100.00 \quad 200.00 \quad 300.00 \quad 400.00 \quad 500.00$

\begin{tabular}{llllll}
\hline 0.0005 & 0.009 & 0.025 & 0.045 & 0.066 & 0.089 \\
0.0010 & 0.020 & 0.052 & 0.089 & 0.128 & 0.168 \\
0.0020 & 0.039 & 0.097 & 0.159 & 0.223 & 0.288 \\
0.0050 & 0.078 & 0.179 & 0.285 & 0.393 & 0.503 \\
0.0100 & 0.111 & 0.249 & 0.391 & 0.536 & 0.682 \\
0.2000 & 0.141 & 0.311 & 0.485 & 0.662 & 0.841 \\
0.0500 & 0.168 & 0.367 & 0.571 & 0.778 & 0.986 \\
0.1000 & 0.179 & 0.391 & 0.608 & 0.827 & 1.048 \\
0.2000 & 0.184 & 0.403 & 0.627 & 0.854 & 1.082 \\
1.0000 & 0.187 & 0.410 & 0.639 & 0.871 & 1.104 \\
& & & & & \\
\hline
\end{tabular}

Fraction of $\mathrm{d} E / \mathrm{d} x$ due to energy losses greater than $E_{\mathrm{patr}}$

\begin{tabular}{cccccc}
$\frac{E_{\text {patr }}}{E_{\mu}} / E_{\mu}$ & 100.00 & 200.00 & 300.00 & 400.00 & 400.00 \\
\hline & & & & & \\
\hline 0.0005 & 0.953 & 0.939 & 0.930 & 0.924 & 0.920 \\
0.0010 & 0.894 & 0.872 & 0.860 & 0.853 & 0.848 \\
0.0020 & 0.790 & 0.764 & 0.752 & 0.745 & 0.740 \\
0.0050 & 0.584 & 0.564 & 0.554 & 0.549 & 0.545 \\
0.0100 & 0.406 & 0.394 & 0.388 & 0.385 & 0.382 \\
0.0200 & 0.247 & 0.243 & 0.241 & 0.240 & 0.239 \\
0.0500 & 0.104 & 0.106 & 0.107 & 0.107 & 0.107 \\
0.1000 & 0.045 & 0.048 & 0.050 & 0.050 & 0.051 \\
0.2000 & 0.017 & 0.018 & 0.019 & 0.019 & 0.020 \\
1.0000 & 0.000 & 0.000 & 0.000 & 0.000 & 0.000 \\
& & & & & \\
\hline
\end{tabular}

TABLE 5

Straggling for $500 \mathrm{GeV}$ muons in iron from direct pair production.

\begin{tabular}{|c|c|c|c|}
\hline$E^{\prime}$ & $I R$ & $\%$ of total & $(\mathrm{d} E)$ \\
\hline$\overline{E_{\mu}}$ & $\bar{R}$ & $\begin{array}{l}(\mathrm{d} E / \mathrm{d} x)_{\text {11 }} \text { with } \\
E^{\prime}>\text { this value }\end{array}$ & $\mathrm{d} x ?$ \\
\hline
\end{tabular}

\begin{tabular}{llll}
\hline 0.0005 & 0.00186 & 0.917 & 0.072 \\
0.001 & 0.00353 & 0.845 & 0.141 \\
0.002 & 0.00716 & 0.737 & 0.247 \\
0.005 & 0.0122 & 0.544 & 0.44 \\
0.01 & 0.0187 & 0.383 & 0.60 \\
0.02 & 0.0264 & 0.240 & 0.74 \\
0.05 & 0.0375 & 0.109 & 0.88 \\
0.1 & 0.0455 & 0.052 & 0.93 \\
0.2 & 0.053 & 0.021 & 0.96 \\
1.0 & 0.0645 & 0 & 0.98 \\
& & & \\
\hline
\end{tabular}

\section{Conclusions}

What is a safe fraction of the $(\mathrm{d} E / \mathrm{d} x)_{\mathrm{dn}}$ to use in shielding calculations? We look at table 5, at the

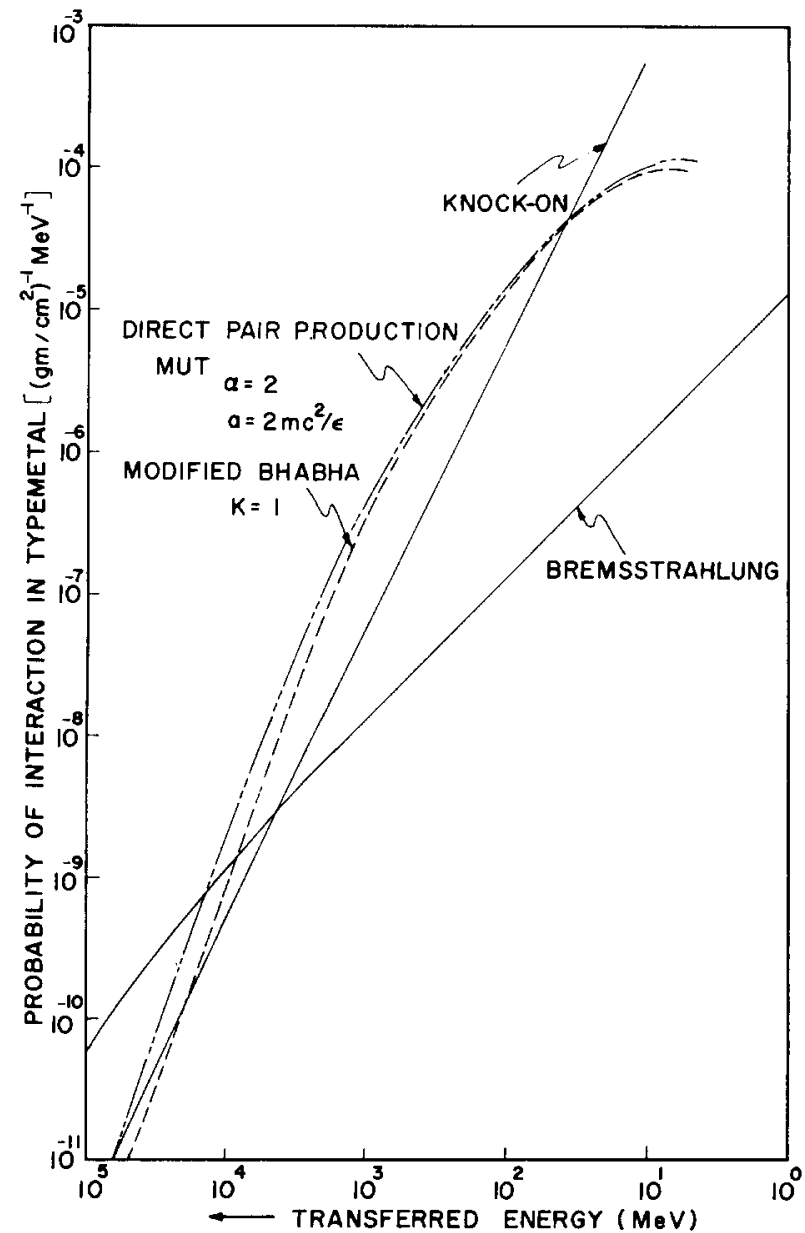

Fig. 1. Direct electron pair production by $200 \mathrm{GeV}$ muons in typemetal $(90 \%$ lead) as a function of energy transferred to the pair.

changes in $\Delta R / R$, i.e. $\Delta(\Delta R / R)$. We ask where $\Delta(\Delta R / R)$. $7 \cdot \overline{(\mathrm{d} E / \mathrm{d} x})$ is equal to the $\Delta(\overline{\mathrm{d} E / \mathrm{d} x)}$ from row to row. $E_{\mathrm{MAX}}^{\prime} / E_{\mu}=0.05$ seems about best. $7 \cdot 0.0375 \cdot(1 / 1.20)$. $(3.28 / 0.98)=0.73$. We then include $11 \%$ loss with energy transfers $>0.05$. Hence for shielding it would appear safe to use

$$
\left(\frac{\mathrm{d} E}{\mathrm{~d} x}\right)_{\text {full collision loss }} 0.79+\left(\frac{\mathrm{d} E}{\mathrm{~d} x}\right)_{\text {full dircet pat ir lows }} 0.52 .
$$

The above fractions should be about right independently of whether Theriot or I have the correct direct pair $\mathrm{d} E / \mathrm{d} x$. Furthermore this should hold unchanged for earth since as seen in tables 3 and 4 the percentage of loss due to high energy transfers remains the same as for iron.

Using the present numbers for losses averaged $0-500 \mathrm{GeV}: 2.26 \cdot 0.79+0.98 \cdot 0.52=2.30 \mathrm{MeV} / \mathrm{gcm}^{2}$ 
should be a reasonable $\overline{(\mathrm{d} E / \mathrm{d} x)}$ to use for shielding calculations in iron for shielding down to the $10^{-12}$ level. (Using Theriot values we obtain $2.20 \cdot 0.79+$ $0.74 \cdot 0.52=2.12 \mathrm{MeV} / \mathrm{gcm}^{2}$.) Hence we find with an iron density of 7.87 that $285 \mathrm{~m}(300 \mathrm{~m}$ with Theriot values) of iron is adequate for $500 \mathrm{GeV}$.

For earth we find at $500 \mathrm{GeV}$ using my numbers (Theriot numbers) $\overline{(\mathrm{d} E / \mathrm{d} x)}=2.55 \cdot 0.79+0.574 \cdot 0.52=$ $2.31(2.51 \cdot 0.79+0.421 \cdot 0.52=2.20) \mathrm{MeV} / \mathrm{gcm}^{2}$. Hence for density 2 we find $1085(1140) \mathrm{m}$ of earth is adequate for $500 \mathrm{GeV}$.

\section{Appendix}

Numerical evaluation and INTEgRation OF the Murota-Ueda-Tanaka direct pair Production CROSS SECTION

The formula is given by ${ }^{10}$ )

$$
\begin{aligned}
\sigma(\varepsilon, s) \mathrm{d} \varepsilon \mathrm{d} s= & \frac{2}{\pi} \frac{N}{A}\left(\frac{Z r_{0}}{13}\right)^{2} L \frac{1}{\varepsilon} \\
& \times\left[\left\{\left[\left(1+\frac{4}{3} \chi\right) \log \left(1+\frac{1}{\chi}\right)-\frac{4}{3}\right]\right.\right. \\
& \left.-\frac{4}{3}\left(s-s^{2}\right)\left[(1+\chi) \log \left(1+\frac{1}{\chi}\right)-1\right]\right\} \\
& \times\left[\frac{E^{2}+(E-\varepsilon)^{2}}{E^{2}}\right]+\frac{8}{3}\left(s-s^{2}\right) \frac{1}{1+\chi} \frac{E-\varepsilon}{E} \\
& +\left\{\left[\frac{1}{3} \frac{1}{\left(1+\frac{1}{\chi}\right)}+\frac{1}{\chi}-\frac{4}{3} \log \left(1+\frac{1}{\chi}\right)\right]\right. \\
& \left.+\left(s-s^{2}\right)\left[-\frac{4}{3 \chi}+\frac{4}{3} \log \left(1+\frac{1}{\chi}\right)\right]\right\} \\
& \left.\times \chi \frac{\varepsilon^{2}}{E^{2}}\right] \mathrm{d} s \mathrm{~d} \varepsilon \operatorname{per} \operatorname{gcm}^{-2},
\end{aligned}
$$

where

$$
\begin{aligned}
s & =\varepsilon^{-} / \varepsilon, \\
\chi & =A\left(s-s^{2}\right), \\
A & =\frac{\mu^{2}}{m^{2}} \frac{\varepsilon^{2}}{E(E-\varepsilon)}, \\
\varepsilon & =\text { combined energy of pair, } \\
\varepsilon_{-} & =\text {energy of electron, } \\
m & =\text { mass of electron, }
\end{aligned}
$$

$\mu=$ mass of incident particle,

$E=$ energy of incident particle,

$r_{0}=$ classical radius of electron,

$\alpha=$ constant of order magnitude 1 ,

$$
\begin{aligned}
L & =\log \left[\frac{2 \alpha E\left(s-s^{2}\right)}{M c^{2}}\right]-1 \text { for non-screening, } \\
& =\log \left[\alpha 137 Z^{-\frac{1}{3}} \frac{M c^{2}}{m c^{2}}\right] \text { for complete screening, }
\end{aligned}
$$

$M^{2}=m^{2}\left[1+\frac{(1-s) s \varepsilon^{2}}{E(E-\varepsilon)} \frac{\mu^{2}}{m^{2}}\right]$.

This formula is valid for the case in which the incident particle has $\mu \gg m$, that $E-\varepsilon \gg \mu c^{2}, \varepsilon \ll E$ and $\varepsilon \gg m c^{2}$. For evaluation, $Z(Z+1)$ was used instead of $Z^{2}$ in order to get a rough correction for electron contribution. The incident particle was considered to be a muon. The process was considered as either completely screened or completely unscreened, the criterion being that the smallest of the two possible values. for $L$ were chosen unless the smallest value was $<0$ in which case 0 was chosen.

The integrals tend to peak near the lower limit and we integrated $\int_{\varepsilon_{\min }}^{E^{\prime}} \int_{s_{\min }}^{\frac{1}{2}} 2 \sigma \mathrm{d} s \mathrm{~d} \varepsilon$ by using a trapedzoidal rule with varying bin widths. Parameter $(N)=$ $[$ parameter $(N-1)] \cdot[1+\Delta] . \Delta=0.1$ was used. Changing $\Delta$ to 0.05 made about a $\frac{1}{2} \%$ change.

\section{References}

1) B. Rossi, High energy physics (Prentice Hall, New York, 1952) ch. II.

2) R. Sternheimer, Phys. Rev. 103 (1956) 511.

3) Table I gives $12.1 \%$ but this must be corrected for the density effect which decreases the low energy transfers only.

4) M. Mando and L. Ronchi, Jr., Nuovo Cimento 9 (1952) 105; Nuovo Cimento 9(1952) 517; Nuovo Cimento 10 (1952) 1275.

5) P. V. Hayman, N. S. Palmer and A. W. Wolfendale, Proc. Roy. Soc. 275A (1963) 391.

6) J. E. Cousins and W. F. Nash, Advan. Phys. 11 (1962) 349.

7) D. Theriot, NAL Report TM229 (1970).

*) G. Racah, Nuovo Cimento 14 (1937) 93.

9) H. J. Bhabha, Proc. Roy. Soc. A 152 (1935) 559; Proc. Cambridge Phil. Soc. 31 (1935) 494.

10) T. Murota, A. Ueda and H. Tanaka, Progr. Theoret. Phys. 16 (1956) 482.

11) B. Roe, Thesis (Cornell University, 1959). 